\title{
Risk of COVID-19 in Healthcare Workers of the Nephrology Department in a Tertiary Hospital
}

\author{
Borja Quiroga, $M D, P h D^{1^{*}}$, Martín Giorgi $M D^{1}$, Patricia Muñoz Ramos, $M D^{1}$, Nelly Daniela Zurita, $M D^{2}$, \\ José Ramón Villagrasa, $M D^{3}$, Cristina Moratilla, $M D^{4}$, Paloma Sanz, $M D^{5}$ and Guillermina Barril, $M D$, \\ $P h D^{1}$
}

${ }^{1}$ Department of Nephrology, Hospital Universitario de La Princesa, Spain

${ }^{2}$ Department of Microbiology, Hospital Universitario de La Princesa, Spain

${ }^{3}$ Department of Epidemiology and Preventive Medicine, Hospital Universitario de La Princesa, Spain

${ }^{4}$ Clínica Fuensanta, Spain

${ }^{5}$ Nephrology Department, Hospital Ruber Juan Bravo, Madrid, Spain

\begin{abstract}
Introduction and Aim: The COVID-19 pandemic has caused devastating socio-sanitary effects worldwide pointing in healthcare workers. The aim of the present study was to analyze COVID-19 incidence, clinical presentation and treatment in the nephrologist of a tertiary hospital.

Material and Methods: All healthcare workers of the Nephrology Department were included. All of them were tested for SARS-CoV-2 virus by real-time reverse transcription-polymerase chain reaction (rRT-PCR) and for antibodies against this virus (IgG and IgM). Data were collected about symptoms, chest X-ray, prescribed treatments and exposure time. All the variables were compared between COVID-19 infected and non-infected workers.

Results: Thirty workers were included, of which 13 (43\%) had COVID-19 infection. Participation in Emergency on-call shifts was associated to COVID-19 $(p=0.02)$. Among the COVID-19 patients, 7 developed symptoms; the most frequent was fever followed by myalgia. Three patients received hydroxycloroquine, one corticosteroids and 6 azithromycin. The use of azithromycin was associated to fever $(p=0.01)$, dysgeusia $(p=0.03)$, asthenia $(p=0.008)$ and cough $(p=0.03)$. Prescription of hydroxycloroquine was associated to dysgeusia $(p<0.001)$ and cough $(p=0.04)$. Positive rRT-PCR and IgG was associated to participation in on-call shifts.
\end{abstract}

Conclusions: The prevalence of COVID-19 in the Nephrology Department is high and associated to the performance of on-call shifts.

\section{Keywords}

COVID-19, Healthcare workers, Nephrology, SARS-CoV-2, Professional exposure

\section{Introduction}

Severe acute respiratory syndrome coronavirus-2 (SARSCoV-2) causes the disease currently known as corona virus disease-19 (COVID-19), which has impacted the world causing thousands of deaths worldwide [1]. Although the main measures established by the authorities to reduce the expansion of COVID-19 were social-distancing and lockdowns, some workers carrying out occupations considered as essential were requested to keep working. Among them, probably the most exposed population to SARS-CoV-2 is the healthcare professionals [2]. In addition to the clinical consequences of COVID-19, at high-risk professionals have had to face critical situations for the first time in their life with the consequent mental overload leading to high rates of mental disorders [3].
Nephrology healthcare workers have been at the front line during the pandemic. In addition to the clinical management of hospitalized patients, dialysis patients are one of the most at-risk populations [4]. Several immune deficiencies

*Corresponding author: Borja Quiroga MD, PHD, Nephrology Department, Hospital Universitario de La Princesa, Madrid, Spain, Tel: +34915202200

Accepted: November 10, 2020

Published online: November 12, 2020

Citation: Quiroga B, Giorgi M, Ramos PM, et al. (2020) Risk of COVID-19 in Healthcare Workers of the Nephrology Department in a Tertiary Hospital. J Ren Surg 2(2):11-16 
have been demonstrated in patients with chronic kidney disease (CKD), especially in those in dialysis [5]. Furthermore, patients requiring hemodialysis need to attend the hospital three times per week, or even more, to undergo dialysis, thereby raising their risk of suffering from nosocomial infections, including SARS-CoV-2. For these reasons, nephrologists have developed their own protocols for managing patients on renal replacement therapies including the creation of especial units for performing dialysis to infected patients [6]. These high rates of exposure of healthcare workers in nephrology departments have probably increased their risk for developing COVID-19, although no information has been published to date. The aims of the present study were to analyze the impact of COVID-19 among the nephrologists and their clinical outcomes during the pandemic.

\section{Material and Methods}

This is a retrospective study including all the healthcare workers of the Nephrology Department in a Spanish tertiary hospital. All the Nephrology department staff accepted to participate in this study. Medical information was anonymously collected including age and sex, blood group, comorbidities (hypertension, diabetes mellitus, dyslipidemia, hypothyroidism and smoking status) and the use of renin-angiotensin-aldosterone system blockers. To evaluate the exposure of healthcare workers, information was requested about their place of work (hospital or hemodialysis center), department (nephrology, internal medicine or emergency) and section (hospitalization, dialysis, only on-call shifts, inpatient care). The unit (emergency and/or nephrology) and the number of on-call shift hours were also collected. The total number of exposure hours was calculated estimating 7 hours per workday and adding the on-call shift hours.

All of them were tested for SARS-CoV-2 by real-time reverse transcription-polymerase chain reaction ( $R R T-P C R$ ) in throat-swab specimens and for immunoglobulin (Ig) $M$ and IgG antibodies in blood samples (serology test). rRT-PCR was performed at any time during the lockdown in Spain (1st March to 1st May) if the healthcare worker had any symptom or in the first week of May when all the workers at the hospital were tested, while serology tests were carried out only during this last period. SARS-CoV-2 detection was performed with the Cobas $^{\circledR}$ SARS-CoV-2 assay on the cobas $^{\circledR} 6800$ System (Cobas ${ }^{\circledR}$, Roche), which automatically extracts nucleic acids followed by RT-PCR amplification of viral RNA, following manufacturer's instructions. The assay targets a specific gene of SARS-CoV-2 (ORF1a) and a conserved region of pan-Sarbecovirus (E-gene) and provides qualitative results (positive or negative).

Positive and negative controls were included in each run, as well as an internal control into each sample during sample processing. IgM+IgA and IgG antibodies against SARS-COV-2 were determined in serum samples. Samples were incubated 30 minutes at 56 으 for heat-inactivation and evaluated by a commercial ELISA Assay Kit (Vircell Spain S.L.U.), using the recombinant antigens of the SARS-CoV-2 proteins Spike glycoprotein (S protein) and Nucleocapside protein (N Protein). The assay was performed using the Automatic Workstation
DS2 (Dynex Technologies Inc.) in a 96-well micro titer plate according to a protocol including washing steps and incubation cycles and using controls and reagents provided in the ELISA Assay Kit. Optical density was measured at $450 \mathrm{~nm}$, the 92 signal to cut-off ratio was calculated and values were expressed according to manufacturer's protocol.

Healthcare workers were considered to be infected with SARS-CoV-2 if they were positive for viral RNA (rRT-PCR), IgM or IgG at any moment of the study. Information about the following symptoms (fever defined as temperature higher than $37.5^{\circ} \mathrm{C}$, diarrhea, anosmia, dysgeusia, myalgia, dyspnea and cough) was requested from confirmed COVID-19 patients. The presence of infiltrates in the chest X-ray (if available) and treatment for COVID-19 were also collected. The study was approved by the local research ethics committee (registry number 4139).

\section{Statistics}

Values are expressed as mean \pm standard deviation or median (interquartile range) depending on their distribution, tested using the Kolmogorov-Smirnov test. All the included patients were divided into two groups according to the infection status during the pandemic (COVID-19 Infected or non-infected). Comparison of the different collected values between COVID-19 Infected and non-infected healthcare workers were performed using Chi-square or t-test for parametric values and Fisher test or Mann-Whitney test for non-parametric variables. In addition, referred symptoms and their association to the prescribed treatments and positivity of rRT-PCR were evaluated using the same tests explained before. The association of SARS-CoV-2 infection and exposure time of the worker was also assessed. All statistical analyses were performed with SPSS 24.0 (SPSS, Inc., Chicago, Illinois, USA). Graphs in the figures were drawn by Graph Pad Prism 6.0 (Graph Pad Software Inc, San Diego, California, USA). P-values $<0.05$ were considered statistically significant.

\section{Results}

\section{Baseline characteristics}

Thirty healthcare workers were included in this study. Thirteen (43\%) of them had COVID-19. The only factor associated to COVID-19 was working in Emergency on-call shifts ( $p=$ 0.02). There was a trend toward higher number of COVID-19 cases among workers of the Nephrology on-call shifts, although this result did not reach statistical significance $(p=$ 0.06) (Table 1).

\section{Clinical findings and treatment}

Among the COVID-19 patients, 7 (53\%) had at least one symptom. The most frequent symptom was fever in 6 patients (46\%), followed by myalgia in 5 (38\%). Among the symptomatic patients, 3 (43\%) received hydroxycloroquine, 1 (14\%) corticosteroids and $6(86 \%)$ azithromycin. Being symptomatic was associated to the use of azithromycin $(p=0.002)$ and to corticosteroid prescription, although this trend did not reach statistical significance $(p=0.07)$. The use of azithromycin was associated to fever $(0.01)$, dysgeusia $(p=0.03)$, asthenia $(p=$ 
Citation: Quiroga B, Giorgi M, Ramos PM, et al. (2020) Risk of COVID-19 in Healthcare Workers of the Nephrology Department in a Tertiary Hospital. J Ren Surg 2(2):11-16

Table 1: Baseline characteristics.

\begin{tabular}{|c|c|c|c|c|}
\hline & Total $(n=30)$ & $\begin{array}{l}\text { COVID-19 infected } \\
(n=13)\end{array}$ & $\begin{array}{l}\text { COVID-19 non-infected } \\
(\mathrm{n}=17)\end{array}$ & $\mathbf{p}$ \\
\hline Sex (male) (n, \%) & $14(47)$ & $7(54)$ & $7(41)$ & 0.49 \\
\hline Age (years) & $42 \pm 13$ & $40 \pm 15$ & $43 \pm 12$ & 0.65 \\
\hline Hypertension (n, \%) & $4(13)$ & $1(8)$ & $3(18)$ & 0.43 \\
\hline RAASi (n, \%) & $3(10)$ & $0(0)$ & $3(18)$ & 0.11 \\
\hline Diabetes Mellitus (n, \%) & $0(0)$ & $0(0)$ & $0(0)$ & -- \\
\hline Dyslipidemia (n, \%) & $4(13)$ & $1(8)$ & $3(18)$ & 0.42 \\
\hline Smoker (n, \%) & $5(17)$ & $2(15)$ & $3(18)$ & 0.86 \\
\hline Hypothyroidism (n, \%) & $3(10)$ & $1(8)$ & $2(12)$ & 0.71 \\
\hline \multicolumn{5}{|l|}{ Blood group } \\
\hline 0 Positive (n, \%) & $10(33)$ & $5(38)$ & $5(30)$ & \multirow{5}{*}{0.11} \\
\hline 0 Negative $(n, \%)$ & $1(3)$ & $1(8)$ & $0(0)$ & \\
\hline A positive $(n, \%)$ & $12(40)$ & $3(23)$ & $9(53)$ & \\
\hline A negative $(n, \%)$ & $5(17)$ & $4(31)$ & $1(6)$ & \\
\hline B positive $(n, \%)$ & $2(7)$ & $0(0)$ & $2(12)$ & \\
\hline \multicolumn{5}{|l|}{ Working place } \\
\hline Hemodialysis center (n, \%) & $11(37)$ & $3(23)$ & $8(47)$ & \multirow{2}{*}{0.17} \\
\hline Hospital (n, \%) & $19(63)$ & $10(77)$ & $9(53)$ & \\
\hline \multicolumn{5}{|l|}{ Working department* } \\
\hline Internal medicine (n, \%) & $6(20)$ & $4(31)$ & $2(12)$ & \multirow{3}{*}{0.40} \\
\hline Nephrology (n, \%) & $22(73)$ & $8(62)$ & $14(82)$ & \\
\hline Emergency (n, \%) & $2(7)$ & $1(8)$ & $1(6)$ & \\
\hline \multicolumn{4}{|l|}{ Working section* } & \multirow{6}{*}{0.25} \\
\hline Hospitalization (n, \%) & $11(37)$ & $5(38)$ & $6(35)$ & \\
\hline Dialysis (n, \%) & $9(30)$ & $3(23)$ & $6(35)$ & \\
\hline Inpatient care (n, \%) & $3(10)$ & $3(23)$ & $0(0)$ & \\
\hline Only on-call (n, \%) & $1(3)$ & $0(0)$ & $1(6)$ & \\
\hline Various (n, \%) & $6(20)$ & $2(15)$ & $4(23)$ & \\
\hline Nephrology on-call shifts (yes) (n, \%)* & $15(50)$ & $9(70)$ & $6(35)$ & 0.06 \\
\hline Emergency on-call shifts (yes) $(n, \%)^{*}$ & $6(20)$ & $5(38)$ & $1(6)$ & 0.02 \\
\hline No. of on-call shift hours & $158 \pm 81$ & $167 \pm 73$ & $150 \pm 94$ & 0.66 \\
\hline Exposure time (weeks) ${ }^{+}$ & $7 \pm 2$ & $8 \pm 1$ & $7 \pm 3$ & 0.23 \\
\hline Total exposure (hours) & $237(179-278)$ & $240(187-278)$ & $237(121-288)$ & 0.55 \\
\hline
\end{tabular}

Values are expressed as mean \pm standard deviation or median (interquartile range).

RAASI: Renin-Angiotensin Aldosterone System Inhibitors; COVID-19: Corona virus disease-19.

*During COVID-19 pandemic. ${ }^{+}$Between $1^{\text {st }}$ of March and $1^{\text {st }}$ of May (maximum 9 weeks).

0.008 ) and cough $(p=0.03$ ) (Table 2 ). Similarly, prescription of hydroxycloroquine was associated to dysgeusia $(p<0.001)$ and cough $(p=0.04)$ (Table 2$)$.

\section{Infection status}

Infection was assessed using PCR and SARS-CoV-2 serology (IgM and IgG). Five (38\%) patients had a positive rRT-PCR, $10(77 \%)$ IgM and 10 (77\%) IgG. In the rRT-PCR positive patients, the mean duration of positivity was $11 \pm 3$ days. Having a positive $\mathrm{rRT}-\mathrm{PCR}$ and IgG was associated to more on-call shift hours, as shown in Figure 1.

Table 3 shows that the symptoms related to a positive rRT-PCR test were dysgeusia $(p=0.01)$, asthenia $(p=0.02)$ and cough $(p=0.01)$.

\section{Discussion}

The main findings of the present study were the high incidence of COVID-19 in nephrologists and the relation between 
Citation: Quiroga B, Giorgi M, Ramos PM, et al. (2020) Risk of COVID-19 in Healthcare Workers of the Nephrology Department in a Tertiary Hospital. J Ren Surg 2(2):11-16

Table 2: Association between symptoms and treatment received.

\begin{tabular}{|c|c|c|c|c|c|c|c|c|c|}
\hline & \multicolumn{3}{|c|}{ Azithromycin } & \multicolumn{3}{|c|}{ Corticosteroids } & \multicolumn{3}{|c|}{ Hydroxycloroquine } \\
\hline & $\begin{array}{l}\text { Yes } \\
(n=6)\end{array}$ & $\begin{array}{l}\text { No } \\
(n=7)\end{array}$ & $\mathbf{P}$ & $\begin{array}{l}\text { Yes } \\
(n=1)\end{array}$ & $\begin{array}{l}\text { No } \\
(n=12)\end{array}$ & p & $\begin{array}{l}\text { Yes } \\
(n=3)\end{array}$ & $\begin{array}{l}\text { No } \\
(n=10)\end{array}$ & $\mathbf{p}$ \\
\hline Fever (n, \%) & $5(83)$ & $1(14)$ & 0.01 & $1(100)$ & $5(41)$ & 0.26 & $2(67)$ & $4(40)$ & 0.41 \\
\hline Diarrhea (n, \%) & $3(50)$ & $1(14)$ & 0.16 & $1(100)$ & $3(25)$ & 0.11 & $2(67)$ & $2(20)$ & 0.12 \\
\hline Myalgia (n, \%) & $4(67)$ & $1(14)$ & 0.05 & $1(100)$ & $4(33)$ & 0.18 & $1(33)$ & $4(40)$ & 0.83 \\
\hline Anosmia (n, \%) & $2(33)$ & $0(0)$ & 0.09 & $0(0)$ & $2(17)$ & 0.65 & $1(33)$ & $1(10)$ & 0.32 \\
\hline Dysgeusia (n, \%) & $3(50)$ & $0(0)$ & 0.03 & $0(0)$ & $3(25)$ & 0.56 & $3(100)$ & $0(0)$ & $<0.001$ \\
\hline Fatigue (n, \%) & $1(17)$ & $0(0)$ & 0.26 & $0(0)$ & $1(8)$ & 0.76 & $1(33)$ & $0(0)$ & 0.05 \\
\hline Asthenia ( $n, \%)$ & $6(100)$ & $2(29)$ & 0.008 & $1(100)$ & $7(58)$ & 0.41 & $3(100)$ & $5(50)$ & 0.11 \\
\hline Cough (n, \%) & $3(50)$ & $0(0)$ & 0.03 & $1(100)$ & $2(17)$ & 0.05 & $2(67)$ & $1(10)$ & 0.04 \\
\hline Pulmonary infiltrate $(n, \%)$ & 2(33) & $0(0)$ & 0.09 & $1(100)$ & $1(8)$ & 0.01 & $1(33)$ & $1(11)$ & 0.32 \\
\hline
\end{tabular}

*Statistic test: Fisher test or Mann-Whitney test.

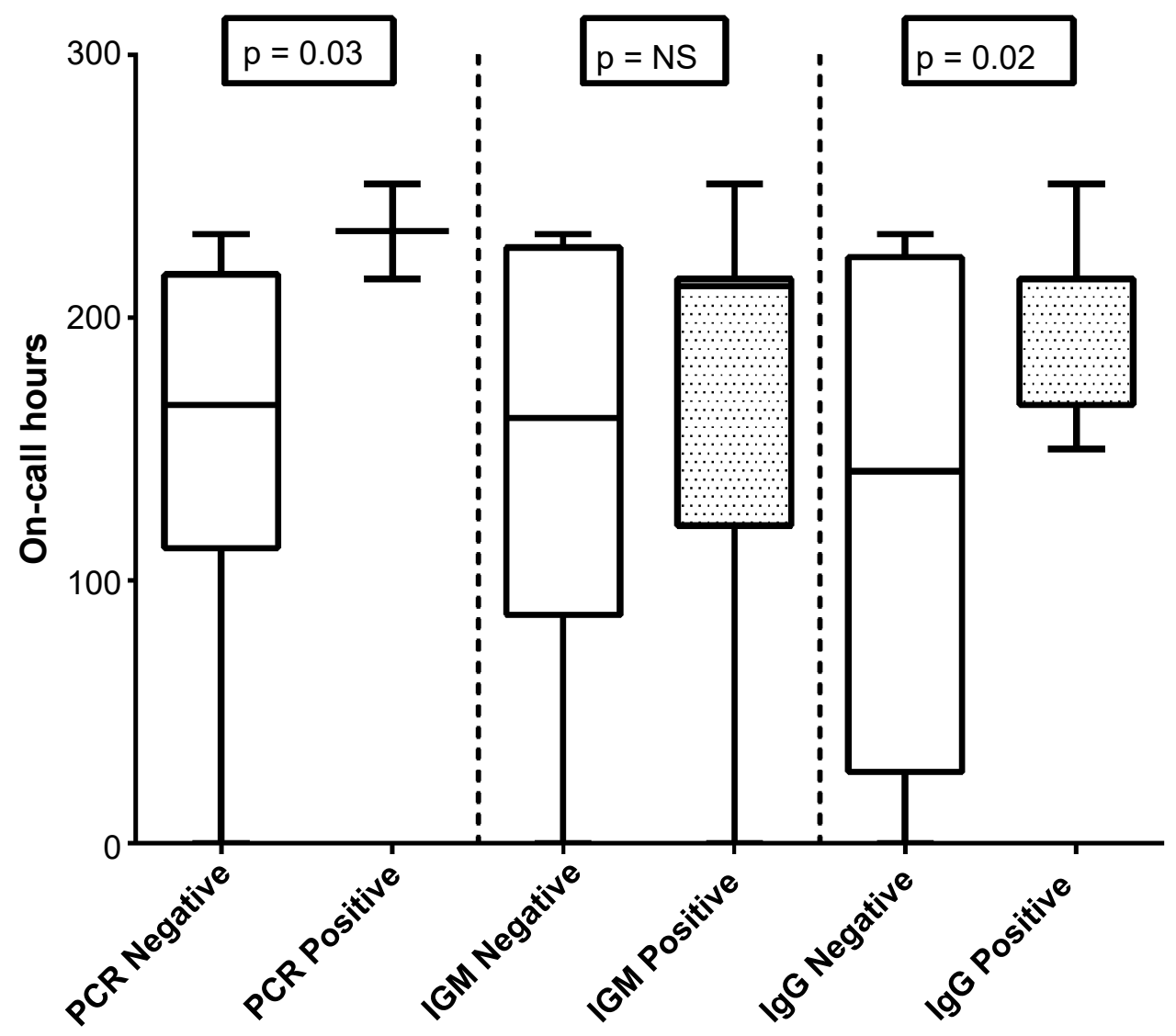

Figure 1: Association between diagnosis of infection and on-call hours.

Abbreviations: NS: Non-Significant; IG: ImmunoGlobulin.

infection and on-call shifts. During the pandemic, specialists have changed their usual roles in clinical practice to provide assistance to patients hospitalized for COVID-19. As a result of this change, the majority of nephrologists have been integrated in other hospital departments especially Internal Medicine, Emergency and Pneumology. In addition, the high rates of SARS-CoV-2 infection in healthcare workers have in- creased sick leave contributing to an elevated workload for those uninfected. In addition to their workday, physicians had to cover for the absence of colleagues, thus multiplying their shifts with the consequent higher exposure to infections. In our department, half of the nephrologists have been doing Nephrology on-call shifts and $20 \%$ of the staff was also required to do Emergency on-call shifts. Our results show that 
Table 3: Association between clinical symptoms and COVID-19 status.

\begin{tabular}{|l|l|l|l|l|}
\hline & $\begin{array}{l}\text { COVID-19 patients } \\
\text { (n=13) }\end{array}$ & $\begin{array}{l}\text { Positive rRT-PCR } \\
\text { (n=5) }\end{array}$ & $\begin{array}{l}\text { Negative rRT-PCR } \\
\text { (n=8) }\end{array}$ \\
\hline Symptoms & $7(53)$ & $5(100)$ & $2(25)$ & 0.08 \\
\hline Fever (n, \%) & $6(46)$ & $4(80)$ & $2(25)$ & 0.05 \\
\hline Diarrhea (n, \%) & $4(31)$ & $3(60)$ & $1(13)$ & 0.07 \\
\hline Myalgia (n, \%) & $5(38)$ & $3(60)$ & $2(25)$ & 0.20 \\
\hline Anosmia (n, \%) & $2(15)$ & $1(20)$ & $1(13)$ & 0.71 \\
\hline Dysgeusia (n, \%) & $3(23)$ & $3(60)$ & $0(0)$ & 0.01 \\
\hline Fatigue (n, \%) & $1(7)$ & $1(20)$ & $0(0)$ & 0.19 \\
\hline Asthenia (n, \%) & $8(62)$ & $5(100)$ & $3(38)$ & 0.02 \\
\hline Cough (n, \%) & $3(23)$ & $3(60)$ & $0(0)$ & 0.01 \\
\hline Chest X-ray infiltrates (n, \%) & $2(15)$ & $2(40)$ & 0.05 \\
\hline
\end{tabular}

Abbreviations: PCR: COVID-19: corona virus disease-19. *Statistic test: Fisher test or Mann-Whitney test.

this increased workload was associated to higher incidence of COVID-19.

The quick spread of SARS-CoV-2 and its virulence have forced the implementation of barrier measures for healthcare workers (personal protective equipment, PPE). However, this equipment has not been always available [7]. Taken together, these two situations have exponentially enhanced the risk of infection in healthcare workers. SARS-CoV-2 infection was detected in 13 nephrologists by rRT-PCR (performed at any moment in symptomatic workers) and by SARS-CoV-2 serology (at the last part of our study). Among the infected patients, $53 \%$ had symptoms and only $38 \%$ had a positive rRTPCR at least once. These results show that during most of the time covered by our study only $53 \%$ of the cases were suspected and only $38 \%$ of them were confirmed. These results have two important consequences. First, since being asymptomatic was initially considered as a criterion of non-transmission, the high proportion of asymptomatic workers could have played a key role in the evolution of the pandemic acting as disease vectors [8]. Second, the late diagnosis could have aggravated late stages of the disease due to the lack of prompt treatment.

COVID-19 treatments against have changed very quickly since the beginning of the pandemic. Local and international protocols have been constantly evolving following the publication of studies that were usually not adequately confirmed or only observational. Accordingly, in our study, nephrologists (sometimes by self-prescription) received a heterogeneous set of drugs driven by different indications. The most frequent treatment was azithromycin followed by hydroxycloroquine. In both cases, dysgeusia and cough were symptoms associated to their use. Surprisingly, the presence of chest X-ray infiltrates did not lead to the prescription of any drug, what was contrary to the recommendations of the clinical guidelines [9]. As none of the 30-studied nephrologists suffered from severe COVID-19, antivirals (such as remdesivir or lopinavir/ritonavir), biological agents or anticoagulation were not used [10-12]. This study has some limitations.
First, the study has a retrospective design. However, all the nephrologists provided full data regarding COVID-19. Second, workday and on-call shifts are not the best markers of exposure, as SARS-CoV-2 infected workers with positive rRTPCR were asked to stay at home for at least 2 weeks. Nevertheless, the association found between on-call shift hours and IgG reduces this limitation, confirming the strong association of exposure and infection. The third limitation is the sample size. Obviously, it is difficult to extrapolate results from a single-center study with 30 workers included. However, the sample is representative as all the nephrologists in the department accepted to participate in the study. In conclusion, nephrologists had an important rate of SARS-CoV-2 infection, although most of them remained asymptomatic.

\section{Acknowlegment}

We want to thank Manuel Gómez Gutierrez for proofreading the manuscript.

\section{Financial Disclosure}

This work has not received any financial support of any kind (public, private or non-profit entity).

\section{Conflict of Interest}

None.

\section{References}

1. Zhu N, Zhang D, Wang W, et al. (2020) A novel corona virus from patients with pneumonia in china, 2019. N Engl J Med 382: 727733.

2. Barranco R, Ventura $F(2020)$ Covid-19 and infection in healthcare workers: An emerging problem. Med Leg J 88: 65-66.

3. El-Hage W, Hingray C, Lemogne $C$, et al. (2020) Health professionals facing the corona virus disease 2019 (COVID-19) pandemic: What are the mental health risks? Encephale 46: S73-S80.

4. Goicoechea M, Sánchez Cámara LA, Macías N, et al. (2020) COVID-19: Clinical course and outcomes of 36 hemodialysis patients in Spain. Kidney Int 98: 27-34. 
5. Hauser AB, Stinghen AE, Kato $S$, et al. (2008) Characteristics and causes of immune dysfunction related to uremia and dialysis. Perit Dial Int 3: S183-S187.

6. de Sequera Ortiz P, Quiroga B, de Arriba de la Fuente G, et al. (2020) Protocol against corona virus diseases in patients on renal replacement therapy: Dialysis and kidney transplant. Nefrologia 40: 253-257.

7. (2020) COVID-19: Protecting health-care workers. Lancet 395: 922.

8. Xiao N, Abboud S, McCarthy DM, et al. (2020) Incidentally discovered COVID-19 in low-suspicion patients-a threat to front line health care workers. Emerg Radiol 25: 1-7.
9. Atzrodt CL, Maknojia I, McCarthy R D P, et al. (2020) A Guide to COVID-19: A global pandemic caused by the novel corona virus SARS-CoV-2. FEBS J

10. Khan Z, Karataş Y, Rahman H (2020) Anti COVID-19 drugs: Need for more clinical evidence and global action. Adv Ther 37: 25752579.

11. Beigel JH, Tomashek KM, Dodd LE, et al. (2020) Remdesivir for the treatment of Covid-19 - preliminary report. N Engl J Med

12. Capra R, De Rossi N, Mattioli F, et al. (2020) Impact of low dose tocilizumab on mortality rate in patients with COVID-19 related pneumonia. Eur J Intern Med 76: 31-35. 